\title{
On the defect pattern evolution in sapphire irradiated by swift ions in a broad fluence range
}

\author{
P.M. Gordo ${ }^{\text {a,* }}$, L. Liszkay ${ }^{\mathrm{b}}$, Zs. Kajcsos $^{\mathrm{b}}$, K. Havancsák ${ }^{\mathrm{c}}$, V.A. Skuratov ${ }^{\mathrm{d}}$, G. Kögel ${ }^{\mathrm{e}}$, P. Sperr $^{\mathrm{e}}$, \\ W. Egger ${ }^{\text {e }}$, A.P. de Lima ${ }^{\text {a }}$, M.F. Ferreira Marques ${ }^{\text {a,f }}$ \\ a ICEMS, Department of Physics, University of Coimbra, P-3004-516 Coimbra, Portugal \\ ${ }^{\mathrm{b}}$ KFKI Research Institute for Particle and Nuclear Physics, P.O. Box 49, H-1525 Budapest 114, Hungary \\ ${ }^{\mathrm{c}}$ Department of Materials Physics, Eötvös University, Pázmány Péter sétány 1A, H-1117 Budapest, Hungary \\ ${ }^{\mathrm{d}}$ Flerov Laboratory of Nuclear Reactions, Center of Applied Physics, JINR, 141980 Dubna, Russia \\ e Institut für Angewandte Physik und Messtechnik LRT2, Fakultät für Luft- und Raumfahrttechnik Werner-Heisenberg-Weg 39, D-85577 Neubiberg, Germany \\ f Instituto Superior Engenharia, P-3031-199 Coimbra, Portugal
}

\section{A R T I C L E I N F O}

\section{Article history:}

Available online $\mathrm{xxx}$

\section{Keywords:}

Sapphire

$\mathrm{Al}_{2} \mathrm{O}_{3}$

Positron lifetimes

Doppler broadening

Defects

Positron trapping

\begin{abstract}
A B S T R A C T
Sapphire samples, irradiated with swift $\operatorname{Kr}(245 \mathrm{MeV})$ ions at room temperature in a broad fluence range, were investigated using a continuous and a pulsed positron beam to study the defect structure created by the passage of the ions in depths of a few micrometers. At small doses, monovacancies were identified as dominant defects and positron trapping centres. These monovacancies are assumed to be highly concentrated inside a cylindrical volume around the ion path with an estimated radius of $\sim 1.5 \mathrm{~nm}$. For higher doses a second type of trapping centre emerges. This second class of structural imperfection was associated with the overlap of the individual ion tracks leading to the formation of larger vacancy clusters or voids.
\end{abstract}

(C) 2008 Elsevier B.V. All rights reserved.

\section{Introduction}

Alumina is a widely used material both as a ceramic and as sapphire single crystal substrate for various industrial applications where the quality of the structure plays a determining role. Consequently, methods to investigate and identify specific structural changes are highly desirable for routine-like technological applications as well. Ion irradiation can induce changes in the microstructure of materials in a controlled way and this helps to clarify structure evolution induced by irradiation.

High-energy heavy ions interacting with material lose their energy through electronic and nuclear processes. Point defects, defect clusters and/or ion tracks can be induced in the irradiated materials up to the penetration depth with defect structure depending on the ion energy, mass and irradiation fluences. In addition to the elastic damage it has been reported that, depending on the material and impinging ion, the electronic stopping above a certain threshold value can also create additional displacement damage in the vicinity of the ion path [1].

\footnotetext{
* Corresponding author. Tel.: +351 239410 629; fax: +351 239829158

E-mail address: pgordo@ci.uc.pt (P.M. Gordo).
}

Positron annihilation spectroscopy (PAS) has been successfully used in studying irradiation-induced defects in different materials. As positrons are uniquely sensitive and selective probes of vacancy type defects, their use can provide important information on the defect structure produced by swift ions. Particularly, the use of slow positron beam allows us to study the defect pattern evolution as a function of ion fluence and/or energy in near surface zones. Despite the numerous experiments reported there are several questions to be resolved regarding the details of defect formation process induced by the impinging swift ions, specifically near to the surface region where the electronic stopping power is a few orders of magnitude higher than the nuclear stopping power. In this region, far from the end of the penetration depth of the implanted ions, it is expected that each ion produces an independent cylindrical volume full of defects around the ion path [1-3]. The aim of this work is to get additional information on the defect production and evolution during and after the highenergy swift heavy ion irradiation process utilizing the high sensitivity of the positron annihilation methods.

\section{Experimental}

$\mathrm{Al}_{2} \mathrm{O}_{3}\left(\begin{array}{llll}0 & 0 & 0 & 1\end{array}\right)$ single crystal samples with a typical thickness of few hundred of micrometers were irradiated at room temperature 
with $245 \mathrm{MeV}$ Kripton ions at the U-400 FLNR JINR cyclotron in

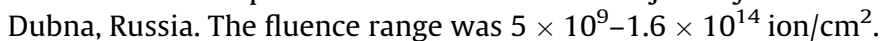
The incident ion beam was perpendicular to the sample surface. To avoid overheating of the irradiated targets the ion flux density was maintained below $2 \times 10^{8} \mathrm{~cm}^{-2} \mathrm{~s}^{-1}$.

In situ positron annihilation Doppler broadening measurements as a function of energy were carried out at room temperature with a magnetically guided slow positron beam in Coimbra [4], whereas the in situ positron lifetime measurements were performed at the pulsed positron beam facility in Munich [5] at $16 \mathrm{keV}$ positron beam energy. These two set-ups were used to characterize the damaged region from the surface up to $\sim 3 \mu \mathrm{m}$ depth.

\section{Results and discussion}

In Fig. 1, the Doppler $S$ parameter values - as a function of positron implantation energy $E$ - are shown for the reference sample and for some irradiated $\alpha-\mathrm{Al}_{2} \mathrm{O}_{3}$ samples. The $S(E)$ parameter values have all been normalized to the bulk value of the reference sapphire sample. As can be observed for all irradiated samples, the $S(E)$ values above $\sim 10 \mathrm{keV}$ of incident positron energy are constant. This behaviour is in agreement with our expectation that near the surface, in the first few $\mu \mathrm{m}$ depth of the material, a homogeneous distribution of defects exists. Each $S(E)$ curve was fitted using the VEPFIT [6] computer code to determine the normalized $S_{\text {sample }}$ value in this region of the irradiated sample (free of surface contribution) and the positron diffusion length $L_{+}$.

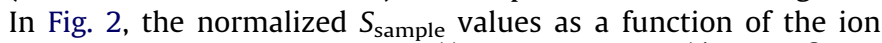
fluence in the range from $10^{11}$ up to $1.6 \times 10^{14} \mathrm{ion} / \mathrm{cm}^{2}$ are presented.

In the fluence range of $10^{11}-10^{13} \mathrm{ion} / \mathrm{cm}^{2}$, the normalized $S$ values do not change indicating that there is a saturation trapping effect. The corresponding Doppler $S$ value is approximately $2.5 \%$ higher than the defect-free bulk and this value can be related to the annihilation of positron in a monovacancy. For higher fluences, the $S$ values increase drastically and a value of $\sim 1.15$ is achieved for a fluence of $1.6 \times 10^{14} \mathrm{ion} / \mathrm{cm}^{2}$. This high $S$ value can only be explained due to the appearance of a new trapping site, i.e., large vacancy clusters or voids, where a longer living trapped positron state can be formed. The increase observed in the positron diffusion length for fluences above $10^{13} \mathrm{ion} / \mathrm{cm}^{2} \quad\left(L_{+}=15 \pm 1\right.$, $39 \pm 1$ and $44 \pm 1 \mathrm{~nm}$ for $10^{13}, 5 \times 10^{13}$ and $10^{14} \mathrm{ion} / \mathrm{cm}^{2}$, respectively) combined with a reduction of the $F^{+}$center ionoluminescence signal produced above the same fluence values [7], indeed strongly

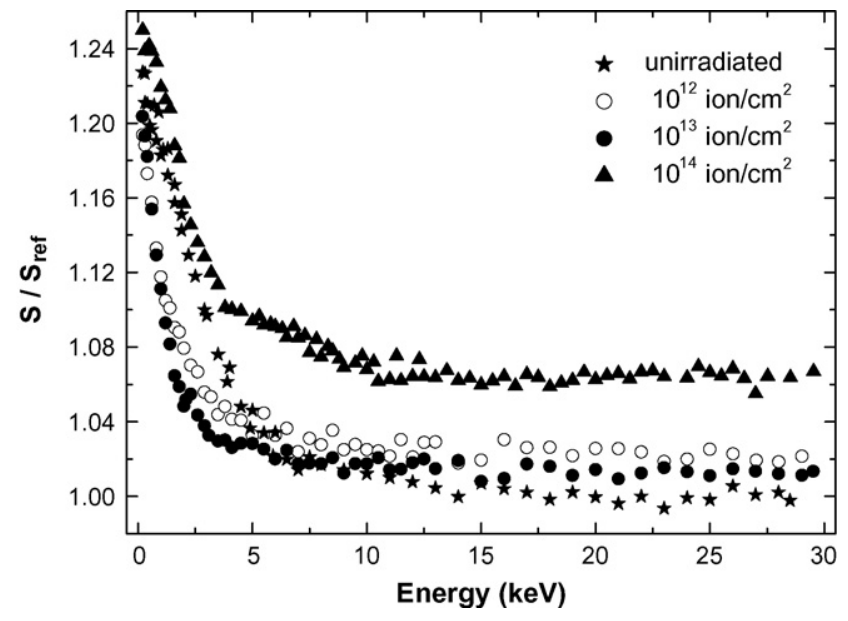

Fig. 1. Doppler $S$ parameter vs. incident positron energy $E$ for the defect-free reference and for the $\mathrm{Kr}$-irradiated sapphire samples.

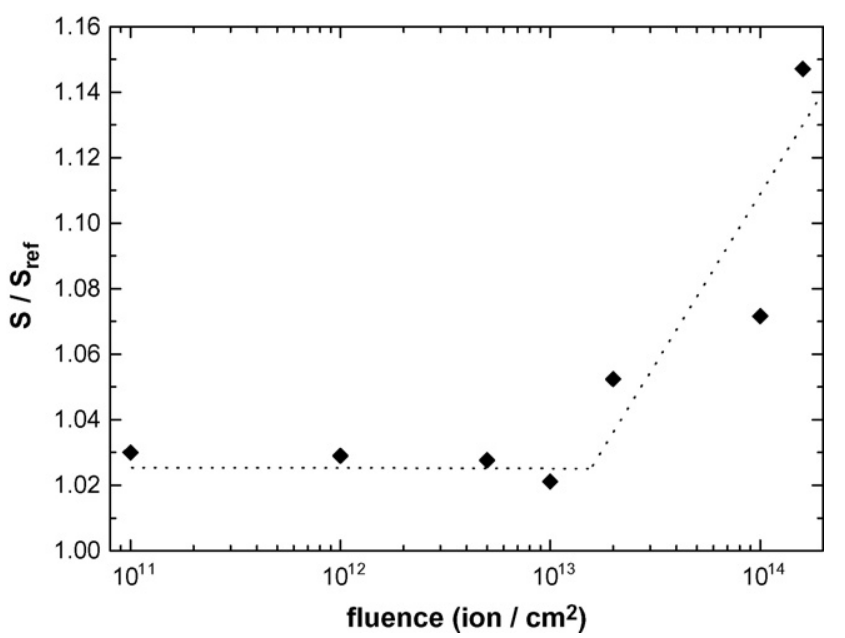

Fig. 2. The $S$ values of irradiated sapphire samples normalized to the defect-free reference sample in the fluence range of $10^{11}-1.6 \times 10^{14}$ ion $\mathrm{cm}^{-2}$. The line is only to guide the eyes.

suggest the clustering of these first kind of traps, i.e., the clustering of monovacancies sets in.

In a previous work [8] the lifetimes related to these two kinds (with differing sizes) of trapping sites induced by the swift ions in sapphire were identified as $186 \mathrm{ps}$ and $500 \mathrm{ps}$, respectively. The creation of the extended positron traps yielding in longer lifetimes was associated with the overlap of individual ion effects. An estimation of the effective region radius can be obtained through the mean distance between the near-neighbour regions. In the simplest geometrical description (one-dimension) we can write the relation between the region radius $r$, the fluence $F$, and the mean distance $d$, as:

$d(\mathrm{~nm}) \approx \frac{10^{7}}{\sqrt{F}-2 r}$

From Fig. 2 it can be seen that the second trapping site appears for fluences above $10^{13} \mathrm{ion} / \mathrm{cm}^{2}$ and assuming $d=0$ at this fluence the use of Eq. (1) provides an upper limit for the region radius $r_{\text {limit }}$ $\sim 1.6 \mathrm{~nm}$.

In order to understand the nature of structural imperfections and their pattern induced by swift ions, a new set of Kr irradiated sapphire samples in the fluence range $5 \times 10^{9}-5 \times 10^{10}$ was used and analysed utilizing the pulsed positron beam at Munich. The sensitivity of Doppler broadening measurements for this fluence range is weak as compared to the lifetime measurement. For each irradiated sample the observed mean lifetime was below $186 \mathrm{ps,}$ the value given above for saturation monovacancy trapping. The analysis of each spectrum indicates that the positron annihilates in two different places, a defect-free sapphire bulk state $\left(\tau_{\mathrm{b}}=145 \mathrm{ps}\right)$ and in a trapped state related to the monovacancy defects $\left(\tau_{\mathrm{d}}=186 \mathrm{ps}\right)$. For example the intensity $I_{2}$ takes values of $37 \pm 1 \%, 64 \pm 1 \%$ and $85 \pm 1 \%$ for fluences of $5 \times 10^{9}, 2 \times 10^{10}$ and $5 \times 10^{10} \mathrm{ion} / \mathrm{cm}^{2}$, respectively.

For the fluence range of this sample set, if it is assumed that the positron trapping sites (monovacancies) are inside a cylindrical volume around the ion path, the radius of the effective ion region can be estimated. In fact, in the vicinity of the surface, far from the projected range of the implanted ions, it is expected that each ion produce an independent cylindrical volume full of defects around the ion track [1-3]. AFM pictures of the damage induced by swift Bi ion irradiation in sapphire support this assumption. Consequently, the positron implanted into the irradiated sapphire sample 


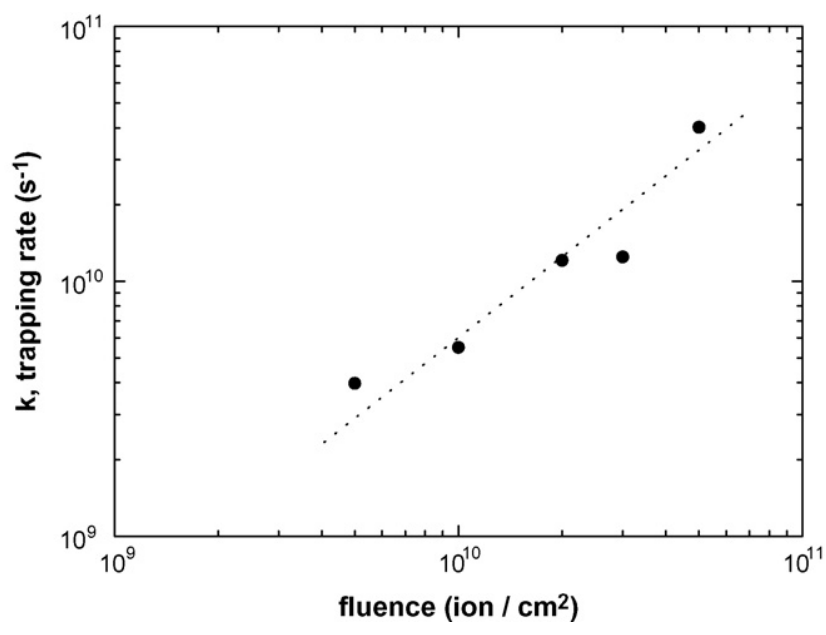

Fig. 3. Overall positron trapping rates in monovacancies as a function of fluence inside the cylindrical volume around the ion track. Fluence range: $5 \times 10^{9}$ $5 \times 10^{10}$ ion $\mathrm{cm}^{-2}$. The line serves only to guide the eyes.

diffuses until the fully defected cylindrical volume around the ion path is reached and than occur the trapping and the annihilation. Assuming this picture, the trapping of positrons is limited by diffusion and the mean distance, $d$, between the border of these cylinders around the two near-neighbour paths, and those parameters should define the trapping rate $k$, of positrons through Eq. (2) as [6]:

$k=\frac{1}{\tau_{\mathrm{b}}}\left[\left(\frac{L_{+}}{d / 2}\right)^{2}-1\right]$

where $\tau_{\mathrm{b}}=145 \mathrm{ps}$ and $L_{+}$represent the positron lifetime and the positron diffusion length, respectively, in the defect-free bulk sapphire sample. The positron diffusion length value, $L_{+}=62 \pm 1 \mathrm{~nm}$ was obtained by the VEPFIT [6] program applied to the $S(E)$ curve of the unirradiated sample shown in Fig. 1.

Fig. 3 shows the experimental values of trapping rate as a function of the ion fluence obtained from the mean lifetime values as [9]:

$k=\frac{1}{\tau_{\mathrm{b}}} \frac{\bar{\tau}-\tau_{\mathrm{b}}}{\tau_{\mathrm{d}}-\bar{\tau}}$

where $\bar{\tau}$ is the mean lifetime of positron in the irradiated sample, $\tau_{\mathrm{d}}=186 \mathrm{ps}$ is the lifetime of positrons trapped in a monovacancy and $\tau_{\mathrm{b}}=145 \mathrm{ps}$ the positron lifetime in the substrate.

Combining Eqs. (1) and (2) and adjusting the values to the experimental data in Fig. 3, the effective radius of the cylindrical volume around the ion track can be estimated. The best agreement is obtained for an effective radius value of $1.5 \mathrm{~nm}$. This value agrees well with the upper limit value of $r_{\text {limit }} \sim 1.6 \mathrm{~nm}$ obtained above when the overlap of tracks was taken into account to explain the creation of extended defects revealed by the second, longer lifetime component.

TRIM code [10] simulations, however, suggest that vacancies created by nuclear collision processes extend over several tens of nanometers around the ion path in the near surface region of $\mathrm{Kr}$ implanted sapphire samples. Thus the small extension (1-2 nm) of the damaged region around the $\mathrm{Kr}$ ion paths estimated by positron annihilation results indicate that these defects might be also formed by inelastic collisions with electrons. It was also observed [1] that collective electronic excitations can create defects in sapphire irradiated with swift ${ }^{238} \mathrm{U}$ ions, and the mean radius of these damage zones was calculated to be between $1.1 \mathrm{~nm}$ and $2.6 \mathrm{~nm}$, depending on the incident ion energy. It has also been shown in ref. [1] that the extended defect formation due to inelastic collisions has a threshold value of $21 \mathrm{keV} \mathrm{nm}^{-1}$. In our case the $(\mathrm{d} E / \mathrm{d} x)_{e}$ value for our $245 \mathrm{MeV} \mathrm{Kr}$ ions was $15.8 \mathrm{keV} \mathrm{nm}^{-1}$, well below the threshold value. This is an apparent contradiction, which might be resolved if we assume that although there is no visible track formation below the threshold value, monovacancies can still be created in the vicinity of the ion path, i.e., ions induce a highly disordered columnar volume, with a radius of $\sim 1.5 \mathrm{~nm}$, surrounded by a larger volume with the presence of monovacancies. The positron lifetime value in this highly disordered region of the bulk material should be quite similar to that in monovacancies, i.e., it is not possible to distinguish between those highly similar defects with positrons. When the ion fluence reaches a critical value that corresponds to the overlap of these highly disordered regions, a second type with a longer living trapped positron state emerges. To better clarify the mechanism discussed here and better understand the defect pattern evolution in sapphire irradiated by swift ions, further positron annihilation measurements are in progress on high energy Bi ion irradiated sapphire samples, where the electronic stopping power values are well above the threshold.

\section{Conclusions}

Positron annihilation measurements on swift heavy ion irradiated samples have identified monovacancies as dominant defects inside the damaged cylindrical volume around the ion path. A value of $\sim 1.5 \mathrm{~nm}$ was calculated for the effective cylinder radius, a value that is in good agreement with the estimation of tracks overlapping above $10^{13} \mathrm{ion} / \mathrm{cm}^{2}$ fluence value. The overlap of cylinders/tracks seems to lead to the aggregation of monovacancies forming large vacancy clusters or voids, revealed by the setting in of a second, longer living trapped positron state.

\section{Acknowledgements}

The support provided by the Hungarian-Portuguese Bilateral Scientific agreement TET No. P23/03 is gratefully acknowledged.

The irradiation in Dubna was supported by a special fund of the Hungarian Academy of Sciences.

The authors are thankful to Dr. G. Huhn for AFM measurements.

\section{References}

[1] B. Canut, A. Benyagoub, G. Marest, A. Meftah, N. Moncoffre, S.M.M. Ramos, F. Studer, P. Thevenard, M. Toulemonde, Phys. Rev. B 51 (1995) 12194.

[2] V.A. Skuratov, S.J. Zinkle, A.E. Efimov, K. Havancsak, Nucl. Instrum. Methods Phys. Res. B 203 (2003) 136.

[3] V.A. Skuratov, A.E. Efimov, D.L. Zagorskii, Phys. Solid State 44 (2002) 165.

[4] A.P. de Lima, C. Lopes Gil, P.M. Gordo, M. Duarte Naia, in: Proc. Workshop on Ion and Slow Positron Beam Utilisation, Costa da Caparica, Portugal, 1998, p. 121

[5] W. Bauer-Kugelmann, P. Sperr, G. Kögel, W. Triftshauser, Mater. Sci. Forum 262365 (2001) 529.

[6] A. van Veen, H. Schut, J. Haakvoort, R.A. Vries, M.R. Ijpma, AIP Conf. Proc. 218 (1990), p. 171.

[7] V.A. Skuratov, private communication.

[8] L. Liszkay, P.M. Gordo, K. Havancsák, V.A. Skuratov, A. de Lima, Zs. Kajcsos, Mater. Sci. Forum 445-446 (2004) 138.

[9] R.N. West, in: P. Hautojärvi (Ed.), Positrons in Solids, Springer, New York, 1979, p. 89.

[10] J.F. Ziegler, J.P. Biersack, U. Littmark, The Stopping and Range of Ions in Solids, Pergamon, New York, 1985. 